\title{
Impact of Adjuvant Therapy Type on Survival in Stage II/III Rectal Cancer Without Preoperative Chemoradiation: A Korean Multicenter Retrospective Study
}

\author{
Byung Mo Kang ${ }^{1}$, Jeong-Heum Baek ${ }^{2}$, Sun Jin Park ${ }^{3}$, Seong Kyu Baek ${ }^{4}$, Ki-Jae Park ${ }^{5}$, Hong-Jo Choi ${ }^{5}$, \\ Byung-Noe Bae ${ }^{6}$, Sun Keun Choi ${ }^{7}$, Kap Tae Kim ${ }^{8}$, Jin-Su Kim ${ }^{9}$, Suk-Hwan Lee ${ }^{10}$ \\ ${ }^{1}$ Department of Surgery, Hallym University Chuncheon Sacred Heart Hospital, Hallym University College of Medicine, Chuncheon; \\ ${ }^{2}$ Department of Surgery, Gachon University Gil Medical Center, Gachon University School of Medicine, Incheon; ${ }^{3}$ Department of Surgery, \\ Kyung Hee University Medical Center, Kyung Hee University School of Medicine, Seoul; ${ }^{4}$ Department of Surgery, Keimyung University \\ Dongsan Medical Center, Keimyung University School of Medicine, Daegu; ${ }^{5}$ Department of Surgery, Dong-A University Hospital, Dong-A \\ University College of Medicine, Busan; ${ }^{6}$ Department of Surgery, Inje University Sanggye Paik Hospital, Inje University School of Medicine, \\ Seoul; ${ }^{7}$ Department of Surgery, Inha University Hospital, Inha University School of Medicine, Incheon; ${ }^{8}$ Department of Surgery, Presbyterian \\ Medical Center, Jeonju; ${ }^{9}$ Department of Surgery, Chungnam National University Hospital, Chungnam National University College of Medicine, \\ Daejeon; ${ }^{10}$ Department of Surgery, Kyung Hee University Hospital at Gangdong, Kyung Hee University School of Medicine, Seoul, Korea
}

Purpose: This study compared the oncologic impact of postoperative chemotherapy and chemoradiotherapy on patients with rectal cancer without preoperative chemoradiation.

Methods: This retrospective study analyzed 713 patients with a mean follow-up of 58 months who had undergone radical resection for stage II/III rectal cancer without preoperative treatment in nine hospitals from January 2004 to December 2009. The study population was categorized a chemotherapy group $(C G, n=460)$ and a chemoradiotherapy group (CRG, $\mathrm{n}=253$ ). Five-year overall survival (OS) and disease-free survival (DFS) were analyzed, and independent factors predicting survival were identified.

Results: The patients in the CRG were significantly younger $(\mathrm{P}<0.001)$ and had greater incidences of low rectal cancer $(\mathrm{P}<$ $0.001)$ and stage III disease $(\mathrm{P}<0.001)$. Five-year OS $(\mathrm{P}=0.024)$ and DFS $(\mathrm{P}=0.012)$ were significantly higher in the CG for stage II disease; however, they were not significantly different for stage III disease. In the multivariate analysis, independent predictive factors were male sex, low rectal cancer and stage III disease for OS and male sex, abdominoperineal resection, stage III disease and tumor-positive circumferential margin for DFS. However, adjuvant therapy type did not independently affect OS (hazard ratio [HR], 1.243; 95\% confidence interval [CI], 0.794-1.945; P = 0.341) and DFS (HR, $1.091 ; 95 \%$ CI, 0.810-1.470; $\mathrm{P}=0.566$ ).

Conclusion: Adjuvant therapy type did not affect survival of stage II/III rectal cancer patients without neoadjuvant chemoradiotherapy. These results suggest that adjuvant therapy can be chosen based on the patient's condition and the policies of the surgeons and hospital facilities.

Keywords: Rectal cancer; Adjuvant therapy; Chemotherapy; Chemoradiotherapy; Survival

Received: August 2, 2016 - Accepted: September 26, 2017

Correspondence to: Suk-Hwan Lee, M.D.

Department of Surgery, Kyung Hee University Hospital at Gangdong, Kyung Hee University School of Medicine, 892 Dongnam-ro, Gangdong-gu, Seoul 05278, Korea

Tel: +82-2-440-6134, Fax: +82-2-440-6295, E-mail: leeshdr@khu.ac.kr ORCID code: https://orcid.org/0000-0001-6470-8620

(C) 2018 The Korean Society of Coloproctology

This is an open-access article distributed under the terms of the Creative Commons Attribution NonCommercial License (http://creativecommons.org/licenses/by-nc/4.0) which permits unrestricted noncommercial use, distribution, and reproduction in any medium, provided the original work is properly cited.

\section{INTRODUCTION}

The standard treatment, under an exact oncologic principle, to cure a patient with rectal cancer is a radical resection with total mesorectal excision. A multimodal strategy with perioperative chemotherapy and radiotherapy might improve survival outcomes. Unlike colon cancer for which adjuvant therapy is recommended for patients with stage III and high-risk stage II disease 
$[1,2]$, an optimal sequence for multidisciplinary treatment of patients with stage II/III rectal cancer has not yet been established, although adjuvant radiotherapy with chemotherapy is recommended after surgery [3]. The oncologic effect of neoadjuvant radiotherapy and the superior local control of concurrent chemoradiotherapy (CCRT) for the treatment of patients with locally advanced rectal cancer have been established $[4,5]$. However, insufficient studies have been conducted on the oncologic benefits of combining chemotherapy and radiotherapy compared with chemotherapy alone in a postoperative adjuvant setting. Although the clinical recommendation for patients with stage II/III rectal cancer is preoperative CCRT, not all patients receive preoperative CCRT. The necessity of preoperative CCRT for the treatment of patients with upper rectal cancer is still under debate. In these circumstances, many clinicians have difficulty deciding on postoperative treatment, especially for patients with circumferentialmargin-negative stage II/III rectal cancer. The aim of this study was to identify survival differences for patients receiving chemotherapy alone or chemoradiation as an adjuvant therapy for stage II/III rectal cancer.

\section{METHODS}

This study was a multicenter, retrospective analysis. Records from January 2004 to December 2009 were reviewed for patients who had undergone radical surgery for pathological stage II/III rectal cancer at 9 hospitals in Korea. All were followed postoperatively for more than 12 months. The definition of rectal cancer was an adenocarcinoma within $15 \mathrm{~cm}$ of the anal verge. Exclusion criteria were (1) neoadjuvant radiation with or without chemotherapy, (2) 30-day mortality, (3) other intraabdominal malignancy within the last 5 years, or (4) malignancy associated with familial adenomatous polyposis or hereditary nonpolyposis colorectal cancer. The reason patients did not undergo neoadjuvant radiation was patient refusal in most cases and was unclear in other cases. Records for 823 patients with rectal cancer were collected from the 9 hospitals. After the data had been reviewed, 110 patients were excluded: 56 did not receive any adjuvant therapy, 6 received radiotherapy without concurrent chemotherapy and 48 were lost to follow-up.

This multicenter, retrospective study was approved by the Institutional Review Board at each hospital. Data were collected from a prospective patient registry or a review of hospital charts and accumulated through a newly designed case-reporting form. The following variables were recorded: (1) baseline characteristics of the patient and the tumor (sex, age, body mass index, American Society of Anesthesiologists physical status classification, longitudinal and circumferential location of tumor); (2) treatment and pathological data (operation date, operation name, $\mathrm{pT}$ category, $\mathrm{pN}$ category, number of harvested lymph nodes, status of circumferential resection margin [CRM], tumor differentiation, and type of adjuvant therapy); and (3) oncologic data (recurrence, date of recurrence, death, and date of last follow-up).

Longitudinal locations of tumors were stratified by distance to the anal verge into low rectal cancer within 0 to $5.0 \mathrm{~cm}$, mid rectal cancer within 5.1 to $10.0 \mathrm{~cm}$ and upper rectal cancer within 10.1 to $15.0 \mathrm{~cm}$. Circumferential locations of tumors were classified into four quadrants as anterior (12 oclock), right lateral (9 oclock), posterior (6 oclock) or left lateral (3 oclock). Circumferential tumors were defined as those that were more than $75 \%$ of the circumference of the rectal diameter. Tumor locations were recategorized into 2 distinct positions: anterior for tumors in an anterior and circumferential location and nonanterior for tumors in both lateral and posterior locations. Pathological staging and histological grading of rectal cancers were recorded according to the seventh edition of the American Joint Commission on Cancer Cancer Staging manual, and tumor-positive CRM was defined as a circumferential margin less than $1 \mathrm{~mm}$ from the tumor.

Eligible patients were divided into an adjuvant chemotherapy group (CG) and an adjuvant chemoradiotherapy group (CRG). The CG included patients who had received a chemotherapeutic agent for more than 6 cycles or 3 months. Chemotherapeutic agents were chosen based on the preference and policy of the surgeons or physicians. The CRG included patients who had received external beam radiation with a median dose of 5,040 cGy in conjunction with 5-fluorouracil (5-FU) and leucovorin (LV) as radiosensitizers.

Data were analyzed by using IBM SPSS Statistics ver. 20.0 (IBM Co., Armonk, NY, USA). Comparative analyses were done by using the Student $\mathrm{t}$-test for continuous variables and the chi-square test for noncontinuous variables. Overall survival (OS) and disease-free survival (DFS) were analyzed by using the Kaplan-Meier method, and the groups were compared using log-rank tests. Cox regression analyses were used to identify independent predictive variables for survival. $\mathrm{P} \leq 0.05$ was considered statistically significant.

\section{RESULTS}

The data on 713 patients with rectal cancer, 435 men and 278 women with a mean age of 63.4 years (range, 31-91 years), were analyzed. In about half (47.5\%), the tumor was in the mid rectum, and an abdominoperineal resection (APR) was performed on 105 patients (14.7\%). Table 1 shows the baseline characteristics of the patients and the tumors. Both the CG and the CRG had more men (58.9\% in the CG vs. $64.8 \%$ in the CRG); patients in the CG were significantly older than those in the CRG (64.5 years in the CG vs. 61.3 years in the $C R G, P<0.001)$. The longitudinal locations of the tumors were significantly closer to the anal verge in the CRG than in the CG $(8.8 \mathrm{~cm}$ in the CG vs. $7.2 \mathrm{~cm}$ in the CRG, $\mathrm{P}<0.001$ ). Therefore, an APR was performed more frequently in the CRG, although the difference was not statistically significant (12.4\% in the CG vs. $19.0 \%$ in the CRG, $\mathrm{P}=0.825$ ). The proportion of stage III disease was $48.3 \%$ in the CG and $70.0 \%$ in the 
Table 1. Baseline characteristics

\begin{tabular}{|c|c|c|c|}
\hline Characteristic & $\begin{array}{c}C G \\
(n=460)\end{array}$ & $\begin{array}{c}\text { CRG } \\
(n=253)\end{array}$ & P-value \\
\hline Male sex & $271(58.9)$ & $164(64.8)$ & 0.122 \\
\hline Mean age (yr) & 64.5 & 61.3 & $<0.001$ \\
\hline \multicolumn{4}{|l|}{ Longitudinal location of tumor } \\
\hline Upper & $139(30.2)$ & $39(15.4)$ & $<0.001$ \\
\hline Mid & $214(46.5)$ & $125(49.4)$ & \\
\hline Low & $107(23.3)$ & 89 (35.2) & \\
\hline Mean distance of tumor from AV (cm) & 8.8 & 7.2 & $<0.001$ \\
\hline \multicolumn{4}{|l|}{ Circumferential location of tumor } \\
\hline Anterior position & $286(62.2)$ & $175(69.2)$ & 0.344 \\
\hline Nonanterior position & $146(31.7)$ & $76(30.0)$ & \\
\hline Unknown & $28(6.1)$ & $2(0.8)$ & \\
\hline \multicolumn{4}{|l|}{ Operation } \\
\hline LAR & $363(78.9)$ & $190(75.1)$ & 0.825 \\
\hline APR & $57(12.4)$ & 48 (19.0) & \\
\hline Other & $40(8.7)$ & $15(5.9)$ & \\
\hline \multicolumn{4}{|l|}{ pStage } \\
\hline$\|$ & $238(51.7)$ & $76(30.0)$ & $<0.001$ \\
\hline III & $222(48.3)$ & $177(70.0)$ & \\
\hline Mean number of harvested lymph nodes & 20.0 & 19.2 & 0.374 \\
\hline \multicolumn{4}{|l|}{ CRM status } \\
\hline Positive & $44(9.6)$ & $30(11.9)$ & 0.579 \\
\hline Negative & $355(77.2)$ & $189(74.7)$ & \\
\hline Unknown & $61(13.3)$ & $34(13.4)$ & \\
\hline \multicolumn{4}{|l|}{ Differentiation } \\
\hline $\mathrm{G} 1 / \mathrm{G} 2$ & $398(86.5)$ & $232(91.7)$ & 0.819 \\
\hline G3 & $17(3.7)$ & $9(3.6)$ & \\
\hline Unknown & $45(9.8)$ & $12(4.7)$ & \\
\hline Chemotherapeutic agents & & & - \\
\hline $\mathrm{FL}$ & $128(27.8)$ & - & \\
\hline Capecitabine & $41(8.9)$ & - & \\
\hline UFT/oral 5-FU & $227(49.3)$ & - & \\
\hline FOLFOX/FOLFIRI & $51(11.1)$ & - & \\
\hline FOLFOX/FOLFIRI + biologic agent & $6(1.3)$ & - & \\
\hline Others & $7(1.5)$ & - & \\
\hline
\end{tabular}

Values are presented as number of patients (\%) unless otherwise indicated. CG, chemotherapy group; CRG, chemoradiotherapy group; AV, anal verge; LAR, low anterior resection; APR, abdominoperineal resection; CRM, circumferential resection margin; FL, 5-fluorouracil (5-FU) with leucovorin; UFT, tegafur-uracil; FOLFOX, oxaliplatin, 5-fluorouracil and leucovorin; FOLFIRI, irinotecan, 5-fluorouracil and leucovorin.

CRG, a significant difference $(\mathrm{P}<0.001)$. The groups had similar numbers of harvested lymph nodes, rates of tumor-positive CRM and tumor differentiations. For patients with stage II cancer, the
Table 2. Chemotherapeutic agents used in the adjuvant chemotherapy group

\begin{tabular}{lcc}
\hline Regimen & Stage II $(\mathrm{n}=238)$ & Stage III $(\mathrm{n}=222)$ \\
\hline FL & $68(28.6)$ & $60(27.0)$ \\
Capecitabine & $11(4.6)$ & $30(13.5)$ \\
UFT/oral 5-FU & $149(62.6)$ & $78(35.1)$ \\
FOLFOX/FOLFIRI & $2(0.8)$ & $49(22.1)$ \\
Others & $8(3.4)$ & $5(2.3)$ \\
\hline
\end{tabular}

Values are presented as number of patients (\%).

FL, 5-fluorouracil (5-FU) with leucovorin; UFT, tegafur-uracil; FOLFOX, oxaliplatin, 5-fluorouracil and leucovorin; FOLFIRI, irinotecan, 5-fluorouracil and leucovorin.

most commonly used chemotherapeutic agents were tegafur-uracil or oral 5-FU (62.6\%), followed by 5-FU with LV (28.6\%) and capecitabine monotherapy (4.6\%). For patients with stage III cancer, oral 5-FU (35.1\%) and a FOLFOX or FOLFIRI regimen (22.1\%) were most frequently used (Table 2 ).

Median follow-up was 58 months. The 5-year local recurrences (LRs) for the 2 groups were similar (10.5\% in the CG vs. $14.8 \%$ in the CRG, $\mathrm{P}=0.236)$. For all patients, 5 -year OS ( $90.0 \%$ in the CG vs. $82.9 \%$ in the CRG, $\mathrm{P}=0.011)$ and 5 -year DFS $(72.4 \%$ in the CG vs. $58.1 \%$ in the $C R G, P=0.002$ ) were significantly longer in the CG. In the subgroup analyses, 5-year OS (94.1\% in the CG vs. $85.4 \%$ in the CRG, $P=0.024)$ and 5 -year DFS $(80.8 \%$ in the CG vs. $66.3 \%$ in the $C R G, P=0.012$ ) were significantly higher in the CG for patients with stage II disease. Patients with stage III disease showed no differences in 5 -year OS $(\mathrm{P}=0.324)$ and DFS (P $=0.335)$ (Fig. 1).

In the univariate Cox regression tests, poor OS was associated with male sex, low rectal cancer, stage III disease and adjuvant chemoradiotherapy. In addition, poor DFS was influenced by male sex, low rectal cancer, APR, stage III disease, tumor-positive CRM and adjuvant chemoradiotherapy (Table 3). In the multivariate Cox regression analyses to determine interactions among variables that could affect survival, independent predictive factors for OS were male sex, low rectal cancer and stage III disease. Independent predictive factors for DFS were male sex, APR, stage III disease and tumor-positive CRM. The type of adjuvant therapy was not an independent prognostic factor for either OS (hazard ratio $[\mathrm{HR}], 1.24 ; 95 \%$ confidence interval $[\mathrm{CI}], 0.79-1.95 ; \mathrm{P}=$ 0.341 ) or DFS (HR, 1.09; 95\% CI, 0.81-1.47; P = 0.566) (Table 4). In the CG, LR occurred in 30 patients (6.5\%), systemic recurrence (SR) in 81 patients $(17.6 \%)$ and combined LR and SR in 13 patients (2.8\%). In the CRG, LR occurred in 17 patients (6.7\%), SR in 70 patients $(27.7 \%)$ and combined LR and SR in 13 patients (5.1\%). The pattern of recurrence did not vary between the 2 groups $(\mathrm{P}=0.201)$.

\section{DISCUSSION}

Treatment strategies to improve survival of patients with rectal 
All patients
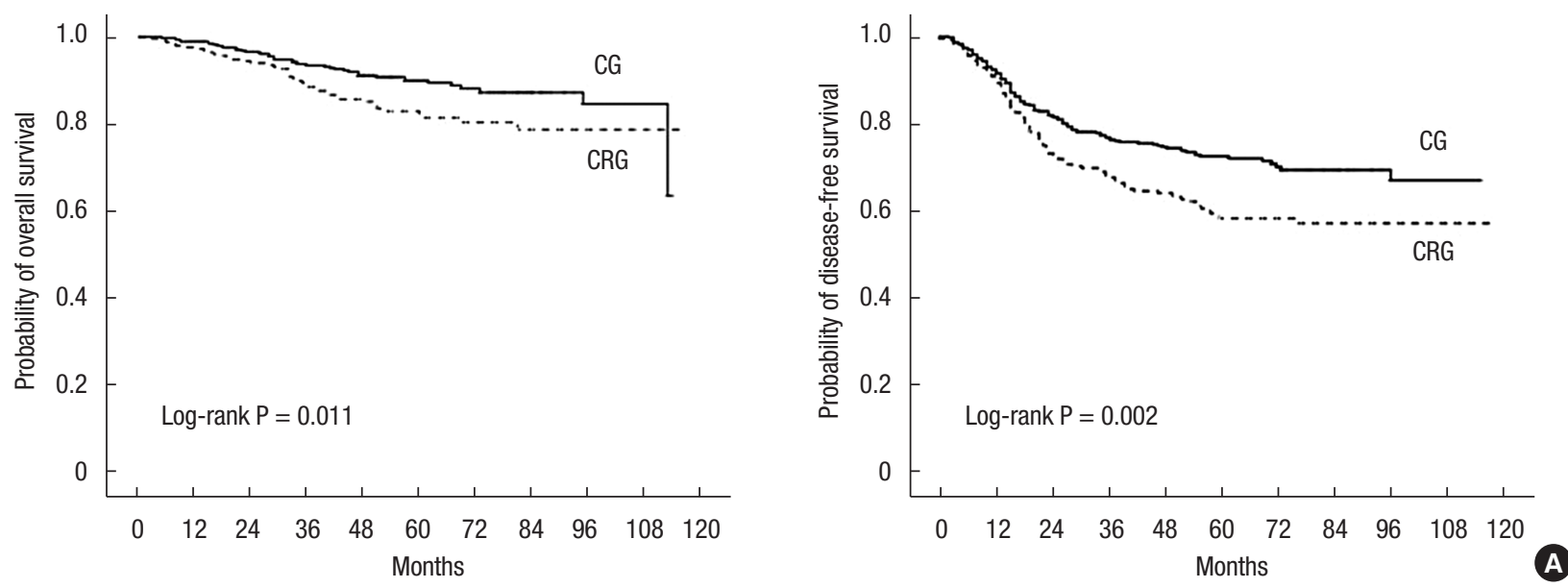

Stage II
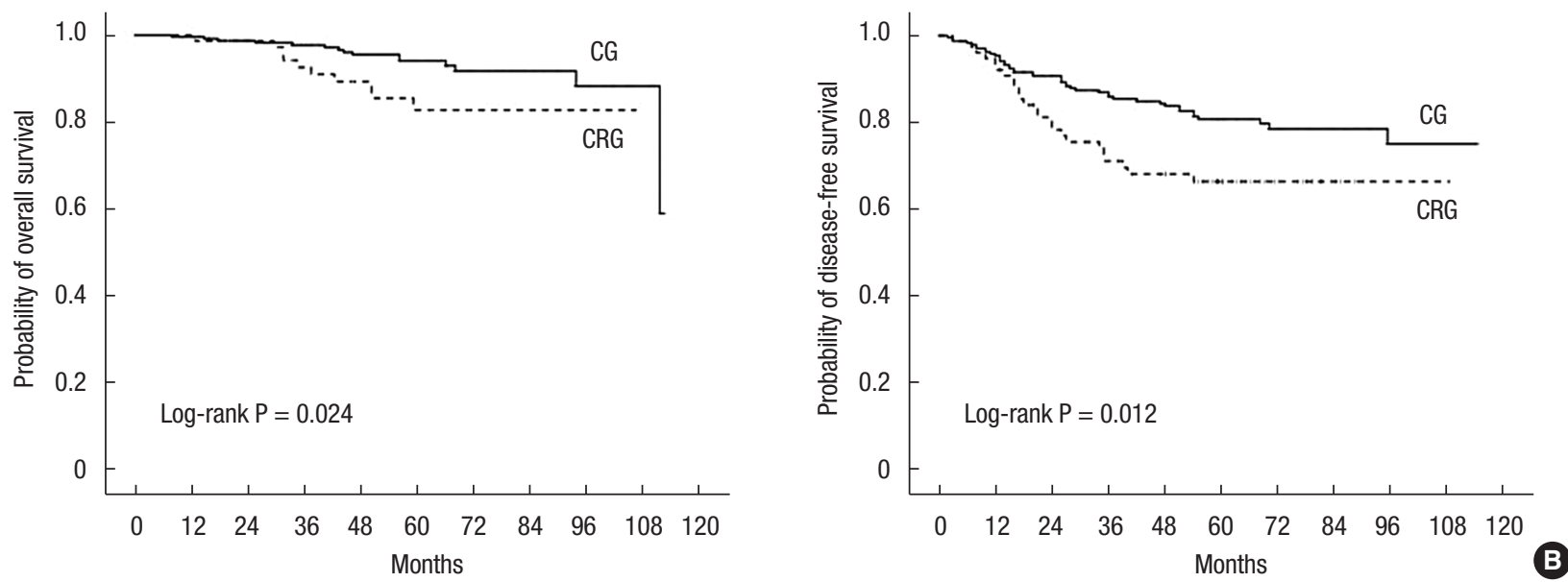

Stage III
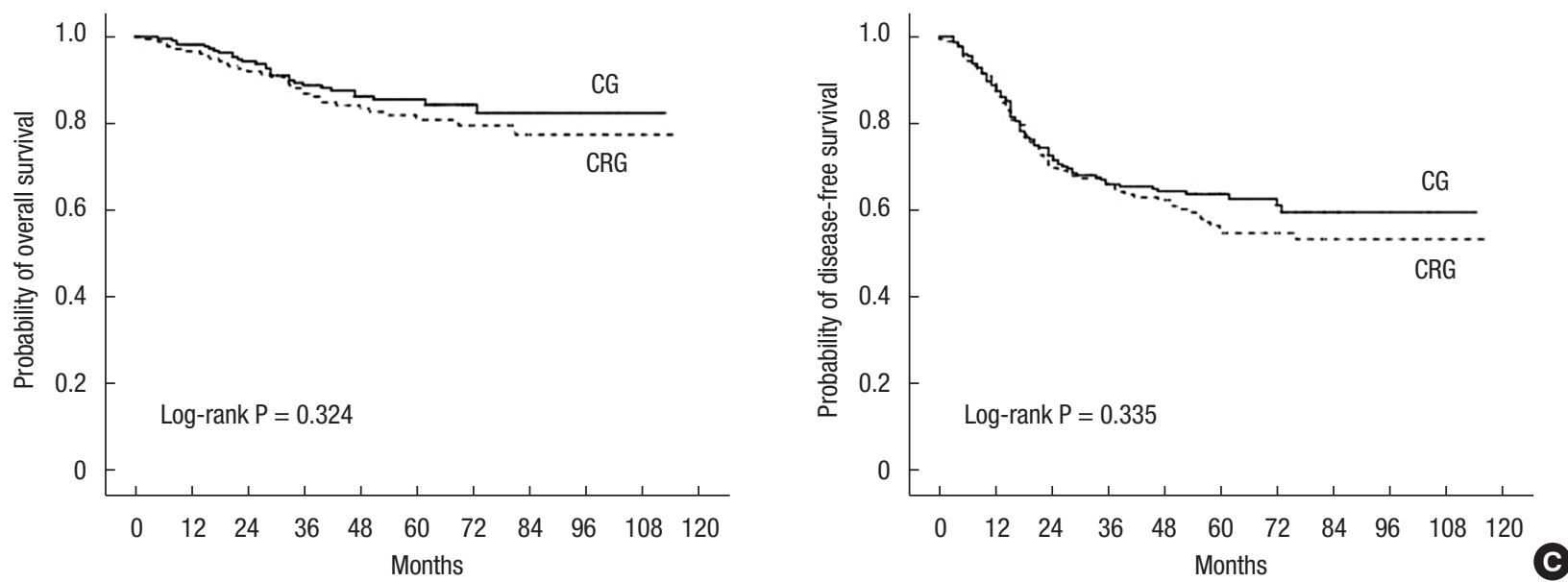

Fig. 1. Probability of overall survival and disease-free survival. All patients (A), stage II (B), stage III (C). CG, chemotherapy group; CRG, chemoradiotherapy group. 


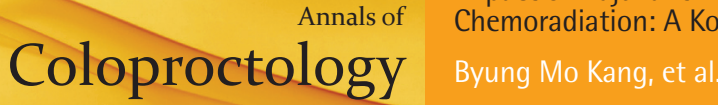

Table 3. Univariate Cox regression analysis of survival

\begin{tabular}{|c|c|c|c|c|c|c|}
\hline \multirow{2}{*}{ Variable } & \multicolumn{3}{|c|}{ OS } & \multicolumn{3}{|c|}{ DFS } \\
\hline & $\mathrm{HR}$ & $95 \% \mathrm{Cl}$ & P-value & HR & $95 \% \mathrm{Cl}$ & P-value \\
\hline Sex & & & 0.021 & & & 0.015 \\
\hline Female & 1 & - & & 1 & - & \\
\hline Male & 1.74 & $1.09-2.77$ & & 1.42 & $1.07-1.87$ & \\
\hline Age (yr) & & & 0.568 & & & 0.153 \\
\hline$\leq 65$ & 1 & - & & 1 & - & \\
\hline$>65$ & 1.13 & $0.74-1.73$ & & 1.21 & $0.93-1.57$ & \\
\hline Longitudinal location & & & 0.046 & & & 0.009 \\
\hline Upper & 1 & - & & 1 & - & \\
\hline Mid & 1.52 & $0.83-2.81$ & 0.177 & 1.20 & $0.85-1.70$ & 0.304 \\
\hline Low & 2.16 & $1.16-4.04$ & 0.016 & 1.70 & $1.18-2.45$ & 0.004 \\
\hline Circumferential location & & & 0.058 & & & 0.152 \\
\hline Nonanterior & 1 & - & & 1 & - & \\
\hline Anterior & 1.65 & $0.98-2.76$ & & 1.24 & $0.92-1.67$ & \\
\hline Operation & & & 0.155 & & & $<0.001$ \\
\hline LAR & 1 & - & & 1 & - & \\
\hline APR & 1.66 & $0.99-2.78$ & 0.054 & 2.02 & $1.47-2.78$ & $<0.001$ \\
\hline Other & 1.07 & $0.46-2.48$ & 0.873 & 1.68 & $1.09-2.61$ & 0.020 \\
\hline Stage & & & 0.002 & & & $<0.001$ \\
\hline$\|$ & 1 & - & & 1 & - & \\
\hline III & 2.08 & $1.31-3.32$ & & 2.05 & $1.54-2.73$ & \\
\hline CRM status & & & 0.572 & & & $<0.001$ \\
\hline Negative & 1 & - & & 1 & - & \\
\hline Positive & 1.21 & $0.62-2.36$ & & 1.93 & $1.34-2.77$ & \\
\hline Differentiation & & & 0.139 & & & 0.473 \\
\hline G1/G2 & 1 & - & & 1 & - & \\
\hline G3 & 1.98 & $0.80-4.91$ & & 1.28 & $0.66-2.49$ & \\
\hline Adjuvant therapy & & & 0.012 & & & 0.002 \\
\hline Chemotherapy & 1 & - & & 1 & - & \\
\hline Chemoradiotherapy & 1.72 & $1.12-2.62$ & & 1.52 & $1.17-1.98$ & \\
\hline
\end{tabular}

OS, overall survival; DFS, disease-free survival; HR, hazard ratio; Cl, confidence interval; LAR, low anterior resection; APR, abdominoperineal resection; CRM, circumferential resection margin.

cancer should be based on a multidisciplinary team approach with radical resection and perioperative radiotherapy and chemotherapy. Especially for T3 or N+ disease, which accounts for $70 \%$ of nonmetastatic rectal cancer $[6,7]$, more precise and aggressive treatment plans are essential to achieve cures. Several studies showed that a radical resection with a total mesorectal excision $[8$, 9] and neoadjuvant chemoradiation [10-13] reduced LR. These treatment methods have been applied in routine clinical practice to patients with rectal cancer.

However, in patients with rectal cancer receiving preoperative radiotherapy, whether additional adjuvant therapy after radical surgery should be given is still debated $[14,15]$. Some studies have investigated the oncologic benefits of postoperative chemotherapy and radiotherapy for patients with rectal cancer. A metaanalysis of 21 randomized trials involving the impact of adjuvant chemotherapy on survival and including 9,785 patients with nonmetastatic rectal cancer showed a significant reduction in risk of death and tumor recurrence in the group that had received postoperative 5-FU-based chemotherapy [16]. In a meta-analysis of patients with rectal cancer done by the Colorectal Cancer Collaborative Group, 5-year local recurrence was significantly lower in the group with vs. without adjuvant radiotherapy (15.3\% vs. 
Table 4. Multivariate Cox regression analysis of survival

\begin{tabular}{|c|c|c|c|c|c|c|}
\hline \multirow{2}{*}{ Variable } & \multicolumn{3}{|c|}{ OS } & \multicolumn{3}{|c|}{ DFS } \\
\hline & $H R$ & $95 \% \mathrm{Cl}$ & P-value & $\mathrm{HR}$ & $95 \% \mathrm{Cl}$ & P-value \\
\hline Sex & & & 0.004 & & & 0.007 \\
\hline Female & 1 & - & & 1 & - & \\
\hline Male & 2.00 & $1.24-3.22$ & & 1.53 & $1.12-2.08$ & \\
\hline Longitudinal location & & & 0.040 & & & \\
\hline Upper & 1 & - & & - & - & - \\
\hline Mid & 1.59 & $0.85-2.95$ & 0.146 & - & - & - \\
\hline Low & 2.25 & $1.19-4.28$ & 0.013 & - & - & - \\
\hline Operation & & & & & & 0.002 \\
\hline LAR & - & - & - & 1 & - & \\
\hline APR & - & - & - & 1.97 & $1.30-3.00$ & 0.002 \\
\hline Others & - & - & - & 1.85 & $1.13-3.04$ & 0.014 \\
\hline Stage & & & 0.003 & & & $<0.001$ \\
\hline$\|$ & 1 & - & - & 1 & - & \\
\hline III & 2.10 & $1.29-3.42$ & - & 2.21 & $1.61-3.03$ & \\
\hline CRM status & & & - & & & 0.006 \\
\hline Negative & - & - & & 1 & - & \\
\hline Positive & - & - & & 1.69 & $1.17-2.44$ & \\
\hline Adjuvant therapy & & & 0.341 & & & 0.566 \\
\hline Chemotherapy & 1 & - & & 1 & - & \\
\hline Chemoradiotherapy & 1.24 & $0.79-1.95$ & & 1.09 & $0.81-1.47$ & \\
\hline
\end{tabular}

OS, overall survival; DFS, disease-free survival; HR, hazard ratio; Cl, confidence interval; LAR, low anterior resection; APR, abdominoperineal resection; CRM, circumferential resection margin.

$22.9 \%, \mathrm{P}=0.002$ ) [17]. The current NCCN guidelines (version 1. 2015) recommend adjuvant chemotherapy or radiotherapy with chemotherapy after radical surgery for patients with locally advanced rectal cancer without neoadjuvant therapy [18].

However, few studies have compared survival benefits between postoperative chemotherapy and chemoradiotherapy in patients with rectal cancer who did not receive preoperative radiotherapy. This study measured the oncologic impact of adjuvant therapy, excluding the tumor-modulating effects of neoadjuvant therapy. We found no difference in OS or DFS according to the type of adjuvant therapy in patients who had undergone radical surgery for stage II/III rectal cancer.

In this study, an APR was performed in only $14 \%$ of the patients, showing that sphincter-saving surgery was more frequent than in trials from Western countries. In the Medical Research Council (MRC) trial of conventional versus laparoscopic-assisted surgery in colorectal cancer (CLASSIC) [19], 27\% of patients in the open surgery group and $25 \%$ in the laparoscopic surgery group underwent an APR. In the colorectal cancer laparoscopic or open resection (COLOR II) trial [20], 23\% in the open surgery group and $29 \%$ in the laparoscopic surgery group underwent an APR. Our tumor-positive CRM rate of $12 \%$ was similar to those found in previous studies. The rates of positive CRM were $14 \%$ in the open surgery group and $16 \%$ in the laparoscopic group in the MRC CLASICC trial [19] and 10\% in the open surgery group and $10 \%$ in the laparoscopic surgery group in the COLOR II trial [20]. Comparative characteristics for the groups showed that the tumors were closer to the anal verge in the CRG than in the CG, probably reflecting that radiotherapy was more frequently applied to patients with mid- to low-rectal cancer than it was to patients with upper rectal cancer. This led to an increased incidence of an APR in the CRG. The proportion of patients with stage III disease was higher in the CRG; therefore, postoperative chemoradiation seemed to be used more often for more advanced rectal cancer.

The chemotherapeutic agents used in the CG were diverse: For stage II disease, oral 5-FU was most commonly used, followed by an intravenous 5-FU/LV regimen. For stage III disease, oral 5-FU was still the most common, but an oxaliplatin-containing or irinotecan-containing regimen and capecitabine were more frequently used than for stage II disease. These trends might be affected by recommendations from the Korean National Health Insurance system. 
Differences in the baseline characteristics that could have affected oncologic outcomes were seen between the groups. Therefore, Cox regression analyses were performed to adjust for compounding variables. In multivariable tests, independent predictive factors for OS were male sex, low rectal cancer and stage III disease; for DFS, those factors were male sex, APR, stage III disease and tumor-positive CRM. However, the type of adjuvant therapy had no statistically significant impact on either OS and DFS.

Previous studies evaluated the survival impact of 2 kinds of adjuvant therapy. In the NSABP Protocol R-01 trial, 555 patients who had undergone radical surgery for rectal cancer were randomly allocated into no further therapy (184 patients), adjuvant chemotherapy with 5-FU, semustine and vincristine (MOF) (187 patients) and adjuvant radiotherapy (184 patients) groups. After a median follow-up period of 64.1 months, adjuvant radiotherapy lowered locoregional failure (21.4\% for chemotherapy vs. $16.3 \%$ for radiotherapy), and distant failure appeared to be lower for chemotherapy ( $24.1 \%$ for chemotherapy vs. $31.0 \%$ for radiotherapy), but these differences were not statistically significant [21]. In the NSABP Protocol R-02 trial, 694 patients who had undergone a radical resection for stage II/III rectal cancer were randomized to receive either adjuvant chemotherapy with MOF or 5-FU/LV (348 patients) or adjuvant radiotherapy with chemotherapy (346 patients). After an average follow-up period of 93 months, radiotherapy with chemotherapy reduced 5-year locoregional relapse from $13 \%$ to $8 \%$ compared with chemotherapy alone. No significant difference was seen in OS $(\mathrm{P}=0.89)$ or DFS $(\mathrm{P}=0.90)$ between the groups [22]. However, MOF combination chemotherapy is not currently recommended, and sequential use of radiotherapy and chemotherapy varied from the currently used treatment sequences. Therefore, the results of these studies should be interpreted with caution with regard to current practice and cannot be directly compared with the result of our study. A recent study by Huh et al. [23] compared postoperative chemotherapy and chemoradiotherapy and found that 5-year DFS (57\% for chemotherapy vs. $55 \%$ for chemoradiation, $\mathrm{P}=0.299$ ) and 5 -year OS (68\% for chemotherapy vs. $63 \%$ for chemoradiation, $\mathrm{P}=0.959$ ) were similar for patients with stage III rectal cancer who had not undergone preoperative chemoradiation.

Our multicenter study primarily targeted the impact of adjuvant chemotherapy and CCRT on the survival of patients with nonmetastatic rectal cancer. Our study had some limitations, such as its retrospective design with potential selection bias. In addition, some patient characteristics were unequally distributed between groups, which might have affected the interpretation of survival outcomes. Furthermore, various chemotherapeutic agents were used for adjuvant chemotherapy, and the oncologic effects of interdrug differences are not known.

The findings of this study suggest that adjuvant CCRT does not have an additional impact on survival for patients with stage II/III rectal cancer compared with chemotherapy alone. Therefore, the choice of adjuvant therapy should depend on the patient's condi- tion and the policies of the surgeons and facilities. In the future, more solid evidence should be generated by prospective trials to overcome the limitations of this retrospective study.

\section{CONFLICT OF INTEREST}

No potential conflict of interest relevant to this article was reported.

\section{ACKNOWLEDGMENTS}

This study was supported by the Chemotherapy Study Group of Korean Society of Coloproctology.

\section{REFERENCES}

1. Figueredo A, Coombes ME, Mukherjee S. Adjuvant therapy for completely resected stage II colon cancer. Cochrane Database Syst Rev 2008;(3):CD005390.

2. Van Cutsem E, Oliveira J; ESMO Guidelines Working Group. Primary colon cancer: ESMO clinical recommendations for diagnosis, adjuvant treatment and follow-up. Ann Oncol 2009;20 Suppl 4:49-50.

3. NIH consensus conference. Adjuvant therapy for patients with colon and rectal cancer. JAMA 1990;264:1444-50.

4. Wong RK, Tandan V, De Silva S, Figueredo A. Pre-operative radiotherapy and curative surgery for the management of localized rectal carcinoma. Cochrane Database Syst Rev 2007;(2):CD002102.

5. McCarthy K, Pearson K, Fulton R, Hewitt J. Pre-operative chemoradiation for non-metastatic locally advanced rectal cancer. Cochrane Database Syst Rev 2012;12:CD008368.

6. Gunderson LL, Jessup JM, Sargent DJ, Greene FL, Stewart AK. Revised TN categorization for colon cancer based on national survival outcomes data. J Clin Oncol 2010;28:264-71.

7. Vignali A, De Nardi P. Multidisciplinary treatment of rectal cancer in 2014: where are we going? World J Gastroenterol 2014;20: 11249-61.

8. MacFarlane JK, Ryall RD, Heald RJ. Mesorectal excision for rectal cancer. Lancet 1993;341:457-60.

9. Enker WE, Thaler HT, Cranor ML, Polyak T. Total mesorectal excision in the operative treatment of carcinoma of the rectum. J Am Coll Surg 1995;181:335-46.

10. Kapiteijn E, Marijnen CA, Nagtegaal ID, Putter H, Steup WH, Wiggers T, et al. Preoperative radiotherapy combined with total mesorectal excision for resectable rectal cancer. N Engl J Med 2001;345:638-46.

11. Bosset JF, Calais G, Mineur L, Maingon P, Radosevic-Jelic L, Daban A, et al. Enhanced tumorocidal effect of chemotherapy with preoperative radiotherapy for rectal cancer: preliminary results-EORTC 22921. J Clin Oncol 2005;23:5620-7.

12. Gérard JP, Conroy T, Bonnetain F, Bouché O, Chapet O, ClosonDejardin MT, et al. Preoperative radiotherapy with or without 
concurrent fluorouracil and leucovorin in T3-4 rectal cancers: results of FFCD 9203. J Clin Oncol 2006;24:4620-5.

13. Swedish Rectal Cancer Trial, Cedermark B, Dahlberg M, Glimelius B, Påhlman L, Rutqvist LE, et al. Improved survival with preoperative radiotherapy in resectable rectal cancer. N Engl J Med 1997;336:980-7.

14. Bujko K, Glynne-Jones R, Bujko M. Does adjuvant fluoropyrimidine-based chemotherapy provide a benefit for patients with resected rectal cancer who have already received neoadjuvant radiochemotherapy? A systematic review of randomised trials. Ann Oncol 2010;21:1743-50.

15. Bosset JF, Calais G, Mineur L, Maingon P, Stojanovic-Rundic S, Bensadoun RJ, et al. Fluorouracil-based adjuvant chemotherapy after preoperative chemoradiotherapy in rectal cancer: long-term results of the EORTC 22921 randomised study. Lancet Oncol 2014;15:184-90.

16. Petersen SH, Harling H, Kirkeby LT, Wille-Jørgensen P, Mocellin S. Postoperative adjuvant chemotherapy in rectal cancer operated for cure. Cochrane Database Syst Rev 2012;(3):CD004078.

17. Colorectal Cancer Collaborative Group. Adjuvant radiotherapy for rectal cancer: a systematic overview of 8,507 patients from 22 randomised trials. Lancet 2001;358:1291-304.

18. National Comprehensive Cancer Network. NCCN Guideline [Internet]. Fort Washington (PA): National Comprehensive Cancer
Network; 2014 [cited 2014 Dec 1]. Available from: http://www. nccn.org/professionals/physician_gls/pdf/rectal.pdf.

19. Guillou PJ, Quirke P, Thorpe H, Walker J, Jayne DG, Smith AM, et al. Short-term endpoints of conventional versus laparoscopicassisted surgery in patients with colorectal cancer (MRC CLASICC trial): multicentre, randomised controlled trial. Lancet 2005; 365:1718-26.

20. van der Pas MH, Haglind E, Cuesta MA, Fürst A, Lacy AM, Hop WC, et al. Laparoscopic versus open surgery for rectal cancer (COLOR II): short-term outcomes of a randomised, phase 3 trial. Lancet Oncol 2013;14:210-8.

21. Fisher B, Wolmark N, Rockette H, Redmond C, Deutsch M, Wickerham DL, et al. Postoperative adjuvant chemotherapy or radiation therapy for rectal cancer: results from NSABP protocol R-01. J Natl Cancer Inst 1988;80:21-9.

22. Wolmark N, Wieand HS, Hyams DM, Colangelo L, Dimitrov NV, Romond EH, et al. Randomized trial of postoperative adjuvant chemotherapy with or without radiotherapy for carcinoma of the rectum: National Surgical Adjuvant Breast and Bowel Project Protocol R-02. J Natl Cancer Inst 2000;92:388-96.

23. Huh JW, Lim SW, Kim HR, Kim YJ. Effects of postoperative adjuvant radiotherapy on recurrence and survival in stage III rectal cancer. J Gastrointest Surg 2011;15:963-70. 